\title{
Gonococci in vivo: Host CMP-NANA, sialylated lipopolysaccharide and serum resistance
}

HARRY SMITH, FRS, (HON)DSC

I N RECENT YEARS THERE HAS BEEN INCREASED INTEREST IN lexamining bacteria obtained directly from patients and infected animals for two reasons: to confirm that putative determinants of virulence indicated by experiments in vitro are, in fact, produced in vivo, and to discover virulence determinants that are induced in vivo but have not yet been seen in vitro. In almost all experiments, however, the in vivo conditions are accepted as a whole. There have been hardly any attempts to dissect out specific host factors that are responsible for the production in vivo of defined virulence determinants. Specification of the influence of factors in the host environment on virulence determinants should be done in future studies on pathogenicity. This paper describes an example of what can be done.

Gonococci obtained directly from patients resist killing by human serum. Most people believe it is important in pathogenicity, and it is of two types. There is a type stable on subculture notably shown by strains from disseminated infection. For most strains, however, (certainly those from urethral pus) there is a rapid loss of resistance. It was not long before we showed that the change was so rapid that it must be a phenotypic change (1). We then went on to show that in human serum and in human red blood cell extracts there was a factor which would induce the gonococcus to resistance. This occurred in vitro if one added the host factor
(2-4). We called it resistance-inducing factor (RIF) and we have been trying to answer the following five questions. First of all, does it appear important in gonorrhea? If it does, what is it? How does it induce resistance? What is the gonococcal determinant concerned? And - the object of the exercise - does it all work in vivo?

In order to answer the first two questions, we needed an assay for RIF. We took serum-susceptible gonococci and induced them to resistance over $3 \mathrm{~h}$ with dilutions of the inducer. We took a small number of these gonococci and incubated them for a short time with either fresh human serum or heated human serum (as controls). Viable counts gave us the percentage resistance of the experimental samples. Plotting percentage resistance against dilution yielded the dilution giving 50\% resistance, which we arbitrarily called 1 RIF unit $(4,5)$.

RIF was important in gonorrhea (6); it was present in male and female genital secretions, in about $20 \%$ human sera, with the proportion greater (31\%) in females infected for the first time (7), in polymorphonuclear and mononuclear phagocyte extracts which are very active (200x red blood cell lysates, 6000x sera) (8) and it affects different gonococcal strains (30 tested). Thirty isolates from Paris venereal disease clinics, in fact, were induced to resistance by RIF. So we had something that looked important. What was it?

From the main talk originally given by Harry Smith (prepared for publication by Dr M Goldner) at a symposium on the Principles of Pathogenicity at the Plenary Session of the International Congress For Infectious Diseases, Montréal, Québec, from July 15 to 20 , 1990. Symposium organized by Biological Implications of Pathogenicity Group in Canada (MG Bergeron and M Goldner presiding)

This work is a part of Professor Smith's close examination of the underlying mechanisms in microbial disease, and illustrates the means used to account fully for microbial pathogenicity 


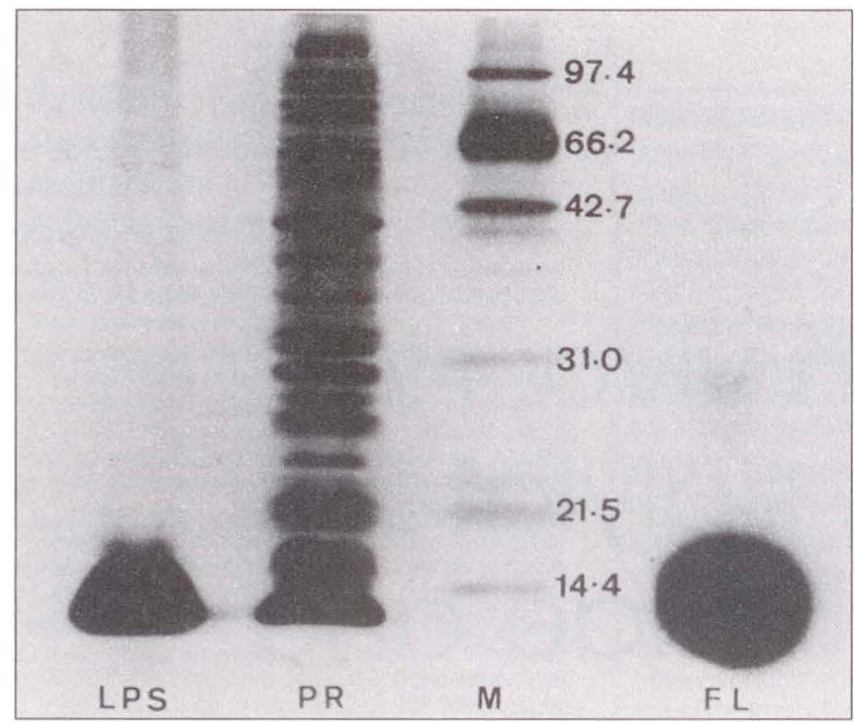

Figure 1) Cytidine 5'-monophosphate-N-acetylneuraminic acid (CMP-NANA)-radiolabelled on NANA moiety to determine if the radiolabel went onto the lipopolysaccharine (LPS). Note, the LPS marker and protein markers $(M)$ indicating the main outer membrane proteins of the gonococcus, which are around $30 \mathrm{kDa}$. A fluorograph of the same gel $(F L)$ is on the right. The radioactivity runs with the LPS. There is a very small amount of radioactivity around $30 \mathrm{kDa}$. PR Protein profile of the resistant organism (silver-stained)

Ultrafiltration showed there was a high $(10,000 \mathrm{kDa})$ and a low (500 to $1000 \mathrm{kDa}$ ) molecular weight RIF. The low molecular weight RIF was extremely acidic and heat-labile. This was a very important property, as will be seen (4). We concentrated on the low molecular weight RIF and fractionated it from blood cell lysates by a process, the details of which have been published (it was really ion exchange by ordinary columns and high performance liquid chromatography (HPLC) (9). We arrived at a single peak. There was not enough material to examine it by conventional analysis (less than a milligram from a litre of blood) so Jim Feeney at the National Institute for Medical Research in London, United Kingdom examined it by nuclear magnetic resonance. He came up with two answers; first, it was not a single component but a mixture and, second, it appeared to contain some pyrimidine nucleotides, which tied in with the ultraviolet absorption spectrum (it had a maximum of $260 \mathrm{~nm}$ ). Coupling this information with the fact that RIF was a very acid-labile compound, we went through lists of pyrimidine nucleotides for an acid-labile one. Christine Nairn found this compound, cytidine 5'-monophospho-N-acetylneuraminic acid (CMP-NANA), a sialylating agent well-known to mammalian biochemists (9). It is acid-labile, and in our resistance induction test, it proved to be fantastically active. Uridine diphosphate(UDP)-N acetyl glucosamine and -galactosamine, two pyrimidine nucleotides that Jim Feeney had actually identified in our final mixture, did not induce resistance because they were acid-
TABLE 1

Neuraminidase restores serum sensitivity

\begin{tabular}{ccc}
\hline $\begin{array}{c}\text { Percentage resistance of gonococci after incubation with: } \\
\text { CMP-NANA then } \\
\text { medium }\end{array}$ & $\begin{array}{c}\text { CMP-NANA then } \\
\text { neuraminidase }\end{array}$ & $\begin{array}{c}\text { Medium then } \\
\text { neuraminidase }\end{array}$ \\
\hline$>95$ & 29 & 4 \\
$>95$ & 37 & 5 \\
$>95$ & 23 & 6 \\
\hline
\end{tabular}

Results are typical of seven results. CMP-NANA Cytidine 5 'monophosphoN-acetylneuraminic acid

\section{TABLE 2}

Gonococci absorb bactericidal antibody

\begin{tabular}{ccc}
\hline Fresh serum & $\begin{array}{c}\text { Fresh serum absorbs } \\
\left.\text { gonococci (at } 4^{\circ} \mathrm{C}\right)\end{array}$ & $\begin{array}{c}\text { Killing test } \\
\text { (\% survival) }\end{array}$ \\
\hline+ & - & $<2$ \\
+ & Susceptible & 77 \\
+ & CMP-NANA & 33 \\
\hline
\end{tabular}

Complement was in excess

stable. UDP and CMP, two other relevant nucleotides, were not active, nor was NANA.

CMP-NANA is a compound of CMP and NANA coupled through the phosphate group. It is a sialylating agent. Under the influence of sialyltransferases, NANA is donated to glycolipids, polysaccharides and glycoproteins in mammalian biochemistry. Could this be our material? Not only was this synthetic material very active in the resistance-inducing test, but for six different criteria (acid lability, heat lability, elution from HPLC and ion exchange chromatography, inhibition by CMP, stability to $\mathrm{N}$-acetyl neuraminidase and change of gonococcal lipopolysaccharide), it was identical to the blood extract preparation. The details have been published and I am just going to mention two of these points $(10,11)$. The fact that CMP inhibited the resistance-inducing activity of both the blood extract preparation and CMP-NANA indicates that sialylation was taking place because it was known that CMP, which is half CMP-NANA, would inhibit the sialylation. The other matter was the change of gonococcal lipopolysaccharide (LPS) (12). It was first indicated by change in pyocin sensitivity pattern. Then, for both blood extract preparation and CMP-NANA-induced resistance the gonococcal LPS separated on SDS-PAGE gels was slow in staining with silver and the final intensity was reduced. Something was happening to the structure $(6,7)$. Now, if you look at this evidence - active synthetic CMP-NANA, six different criteria verifying as identical to blood extract preparation - I think we have the answer to our second question. CMP-NANA is low molecular weight fraction (Mr) RIF. There is less than $50 \mu \mathrm{g} / \mathrm{L}$ blood, so we were very fortunate to identify it. Of course, as we all know, in research you need a bit of luck to make any progress. We certainly had it here.

The next question is: how does CMP-NANA work? We virtually had the answer before we started; probably by 


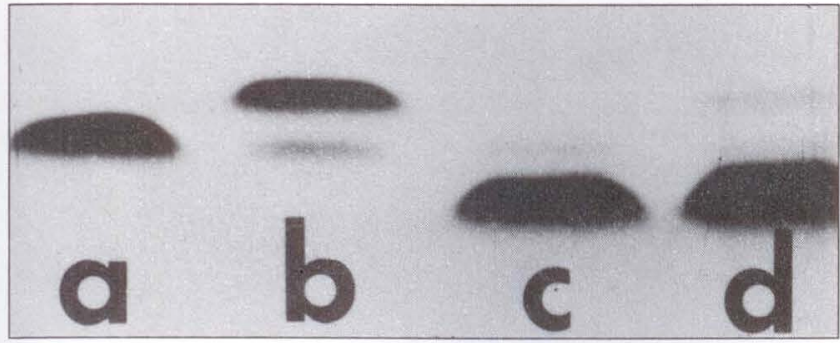

Figure 2) a Silver-stained lipopolysaccharine (LPS) of the parent strain with the terminal group on it. $\mathbf{b}$ Under the influence of cytidine 5'-monophospho-N-acetylneuraminic acid. (CMP-NANA), the molecular weight increases because it is sialylated. A smaller second component in the gel underlying the first is not affected: $\mathbf{c}$ The LPS of the pyocin-selected mutant does not have that terminal group on it; $\mathbf{d}$ The molecular weight of the mutant LPS does not increase under CMP-NANA treatment

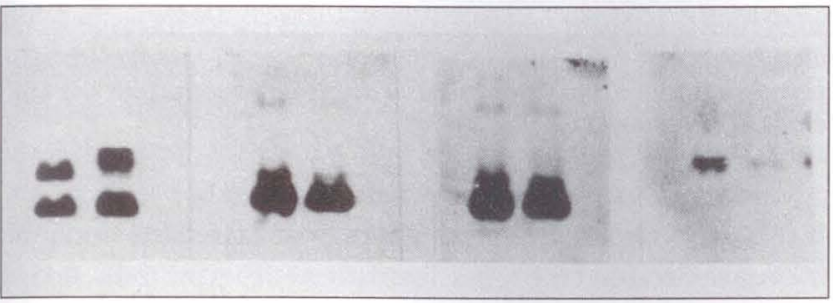

Figure 3) Evidence from monoclonal antibodies. In each case, the tracks on the right show the effect of treatment with cytidine 5 '-monophospho- $N$-acetylneuraminic acid (CMP-NANA). Far left The silver-stained lipopolysaccharine (LPS) gel of a revertant of the previous mutant which made both types of LPS. Centre two panels Blotting with two monoclonal antibodies (MAbs) that recognize epitopes on the main structure, not the terminal group (their reactions are not influenced by incubation with CMPNANA). Right panel Blotting with the MAb that detects the terminal group before treatment with CMP-NANA but not after

sialylating the LPS and inhibiting the reaction with bactericidal immunoglobulin (Ig) M. These are known facts: CMP-NANA is a sialylating agent and LPS is the target for bactericidal IgM. Changes in LPS structure affects this reaction and this, in turn, affects serum sensitivities. In our own particular system, we knew that CMP would inhibit the resistance induction, indicating that sialylation took place. We had already shown that the LPS from our test strain of gonococci would absorb bactericidal IgM and, as I have already indicated, we had a change in LPS structure. So our hypothesis looked good right from the start but we had to prove it.

Obviously, we had to use CMP-NANA radiolabelled on the NANA moiety to see if the radiolabel went on to the LPS $(10,11)$. As shown in Figure 1, radioactivity runs with the LPS. There is a very small amount of radioactivity around $30 \mathrm{kDa}$, but that is not surprising. Penny Hitchcock, some years ago, showed that the principal outer membrane proteins could complex some LPS. If all proteins are removed by proteinase A before SDSPAGE, only the LPS area stains with silver and corresponds with the radioactivity (so radioactivity is on the LPS and nowhere else). But, of course, radioactivity could be transferred as bits and pieces and not as intact NANA. We had to prove that the whole moiety was being transferred. We did this in two ways. CMP inhibited the transfer of radioactivity to the LPS by radiolabelled CMP-NANA. Neuraminidase removed the radiolabel from the sialylated LPS, so there is no doubt that sialylation is taking place $(10,11)$.

But there could be something else - the CMP-NANA might be inducing new gene expression; in particular, it could be inducing a sialyltransferase. We had an idea that this was not so, because the protein profiles of resistant organisms and susceptible organisms were the same, with no new proteins. But we checked by more sensitive pulse-chase experiments. Gonococci were initially inoculated with and without CMP-NANA in the absence of methionine. 35S methionine was added after 5 and 20 mins, followed 10 mins later by a large excess of unlabelled methionine. Radiolabelled proteins from lysed bacteria were detected by fluorography after separation by SDS-PAGE. No differences in the protein profiles of bacteria incubated in the presence or absence of CMP-NANA could be detected. Thus, there was no indication that CMP-NANA activates new gene expression during conversion of susceptible gonococci to resistance. This suggests that the gonococcus has a sialyltransferase. In joint work that I am going to describe, Rob Mandrell at San Francisco has some indications, for the first time, that there is a sialyltransferase in the gonococcus (13).

Sialylation takes place, but is it responsible for resistance? We had the evidence one way round; put the sialyl groups on the LPS and resistance is induced. Could we do it the other way round (take off the sialyl groups with neuraminidase and get back to susceptibility)? It is a tricky experiment because you have got to keep the controls resistant on incubation with medium. We did it, as shown in Table 1 (representative of seven experiments). Gonococci induced to resistance with CMP-NANA were incubated with medium for periods from 40 to 90 mins. There was no reduction in serum resistance. With neuraminidase, there was a gross reduction in resistance. The normal susceptible controls, ie, not incubated with CMP-NANA to induce them to resistance, gave their usual result when incubated with neuraminidase. With removal of the sialyl groups, therefore, there is a gross move to susceptibility from resistance (14).

The other piece of evidence needed to answer our third question was to show that when gonococci became resistant due to sialylation of LPS there was reduced absorption of bactericidal antibody. Fresh serum was absorbed with either susceptible or resistant (CMP-NANA) gonococci and then tested in a normal bactericidal test. The control serum was bactericidal, but there was a gross reduction after absorption with the susceptible organisms (Table 2). This reduction was 

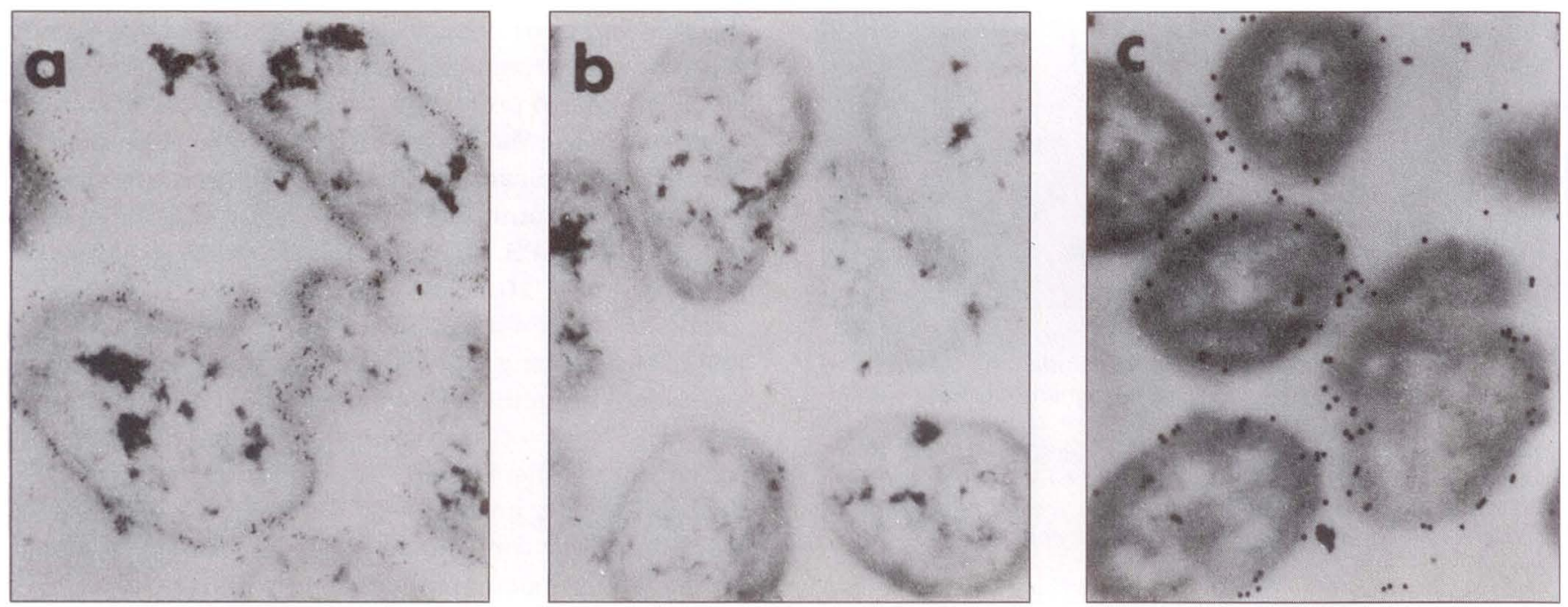

Figure 4) a Strain 1291 viewed by electron microscopy has the conserved component with the terminal group treated with a monoclonal antibody (MAb) that recognizes the group, followed by secondary antibody and small gold particles; $\mathbf{b}$ After cytidine 5'-monophospho$N$-acetylneuraminic acid (CMP-NANA) induction to resistance and similar treatment with gold labelling, there is no reaction as gold particles are not visible; $\mathbf{c}$ If the CMP-NANA-treated organisms are, in turn, treated with neuraminidase and then again with the MAb followed by secondary antibody coupled to larger gold particles, the reaction is restored

much smaller after absorption with the resistant organisms. The answer to our third question is what we thought it would be: sialylation masks LPS target sites for bactericidal antibody, and this is responsible for resistance (11).

The fourth question was what gonococcal determinant was concerned? We knew it was sialylated LPS, but which LPS component and where was the site of sialylation. We needed some help, and we set up some collaborations about two years ago with Rob Mandrell in San Francisco and with Mike Apicella in Buffalo. The experiments that I am going to present are this joint work that has been published (13). The putative structure, which is conserved in over $90 \%$ of gonococci examined and has a molecular weight of around 4.5 $\mathrm{kDa}$, contains a Gal $\beta 14$ Glc NAc group as the terminal disaccharide on its short side chain. The American workers had two tools to look at the involvement of this conserved LPS component in sialylation. First, they had strains that made the component and strains that did not. More specifically, by pyocin selection they had mutants of wild types that made an LPS which was lacking the terminal group. Second, they had monoclonal antibodies that would detect the terminal group and monoclonal antibodies that would detect epitopes an the main structure.

Let us look at some of the experiments. In Figure 2a, the silver-stained LPS of the parent strain (strain 1291 from Mike Apicella) is present with the terminal group on it. Under the influence of CMP-NANA, there is a change in molecular weight - as you would expect, the molecular weight increases because it is sialylated. As shown in Figure 2b, a smaller second component underlying the first is not affected. The LPS of the pyocinselected mutant does not have that terminal group on it (Figure 2c); the molecular weight of the LPS does not increase under CMP-NANA treatment (Figure 2d). A tiny amount of the LPS with the terminal group made by some revertants increases in molecular weight. In a second series of experiments, the LPS of eight strains that made this component of around $4.5 \mathrm{kDa}$ added on radiolabelled NANA from CMP-NANA (radiolabelled), whereas with the LPS of four strains that did not have the conserved $4.5 \mathrm{kDa}$ component, it did not add on the label.

The evidence from monoclonal antibodies is shown in Figure 3; in each of the four panels the tracks on the right show the effect of treatment with CMP-NANA. The left hand panel shows the silver-stained LPS of a revertant of the previous mutant which made both types of LPS, ie, the smaller Mr LPS without the terminal group and the larger Mr LPS with the terminal group. Under the influence of CMP-NANA, the molecular weight of the LPS with the terminal group increases (but not that of the LPS lacking the terminal group). In the two centre panels we have blotting with two monoclonal antibodies (MAbs) that recognize epitopes on the main structure, not the terminal group. Their reactions are not, in fact, influenced by incubation with CMP-NANA. But in the far right panel, the MAb detects the terminal group before treatment with CMP-NANA but not after; hence, the terminal group is masked after sialylation by CMP-NANA. This work was compounded by immunogold labelling on whole organisms. As seen in Figure 4a, strain 1291 has the conserved component with the terminal group treated with a MAb that recognizes the group, followed by secondary antibody and small gold particles. There are the small gold particles around the periphery of the gonococcus. After CMP-NANA induction to resistance and similar treatment with gold labelling, there is no reac- 


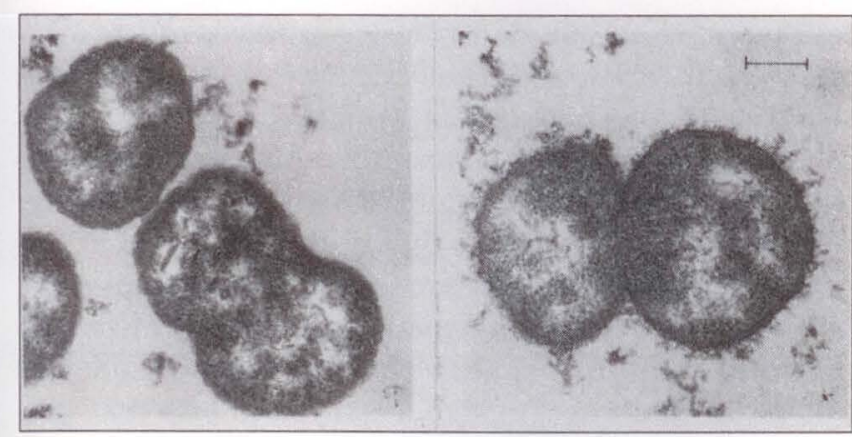

Figure 5) View of gonococci under the electron microscope. After staining the control and cytidine 5'-monophospho- $\mathrm{N}$-acetylneuraminic acid (CMP-NANA)-treated organisms with ruthenium red, the structure formed under the influence of CMP-NANA is very reminiscent of the capsular structure that has been described

tion as gold particles are not visible (Figure $4 \mathrm{~b}$ ). If the CMP-NANA-treated organisms are, in turn, treated with neuraminidase and then again with the MAb followed by secondary antibody coupled to larger gold particles, you will see the reaction restored (Figure 4c). Hence, sialylation had masked the site of reaction of the MAb. Thus, there is little doubt that the gonococcal LPS component involved in sialylation is the $4.5 \mathrm{kDa}$ conserved component and that the site of sialylation is the terminal Gal $\beta 14$ Glc NAc group (13).

When we found the effect of CMP-NANA on the gonococcus, we asked Alan Currie and Andrew Fox at Manchester Public Health Laboratory to have a look at the effect of CMP-NANA on the meningococcus. The meningococcus, of course, is capsulated. They repeated

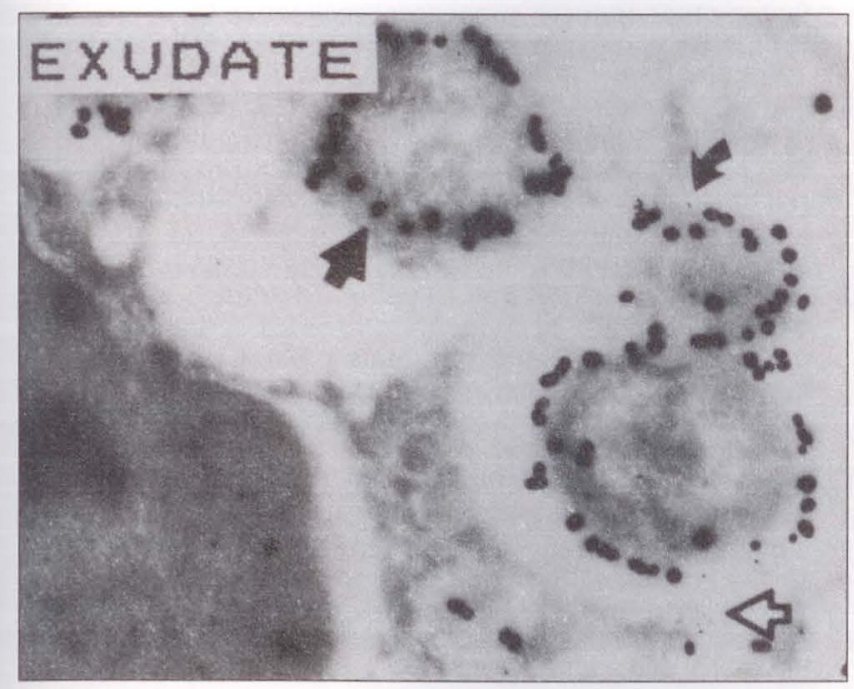

Figure 6a) Control. Results of immunogold labeling experiments with urethral exudate. Very small gold particles (indicated by the open arrows) denote the initial reaction with the monoclonal antibody (MAb); this is minimal in the control. The very large particles (indicated by the straight solid arrows) show that the cocci are gonococci by reaction with a specific antiserum. The intermediate sized particles indicated by the curved solid arrows show the reaction with the MAb after incubation with medium our work on CMP-NANA induction of gonococci to resistance, but in addition they looked at the gonococci under the electron microscope, with dramatic results. After staining the control and CMP-NANA-treated organisms with ruthenium red (which stains acid polysaccharides), the structure formed under the influence of CMP-NANA was very reminiscent of the capsular structure that has been described by some people in the past (Figure 5). The structure is probably sialylated LPS because with neuraminidase treatment it could not be seen. So, we have to fit the surface structure in with the fact that the component of around $4.5 \mathrm{kDa}$ is being sialylated. We are still on with that problem (15-18).

Now the big question: is the LPS of gonococci in vivo sialylated? That is what it is all about and I think the answer is yes, on two pieces of evidence; first, from joint work with Mike Apicella and Bob Mandrell, and second, from some recent work at Birmingham. Let me first remind you that Mike Apicella at Buffalo has a MAb which will detect the terminal Gal $\beta 14$ Glc NAc group on the conserved $4.5 \mathrm{kDa}$ LPS component. If this component is present on gonococci in vivo and the terminal group is sialylated, it will not react with the MAb unless the gonococci are pretreated with neuraminidase. The results of immunogold labeling experiments with urethral exudate are shown in Figure 6. Very small gold particles indicated by the open arrows denote the initial reacton with the MAb; this is minimal in both the control and neuraminidase-treated urethral exudate (Figure 6). The very large particles show that the cocci are, in fact, gonococci by reaction with a specific antiserum. The intermediate sized particles show the reac-

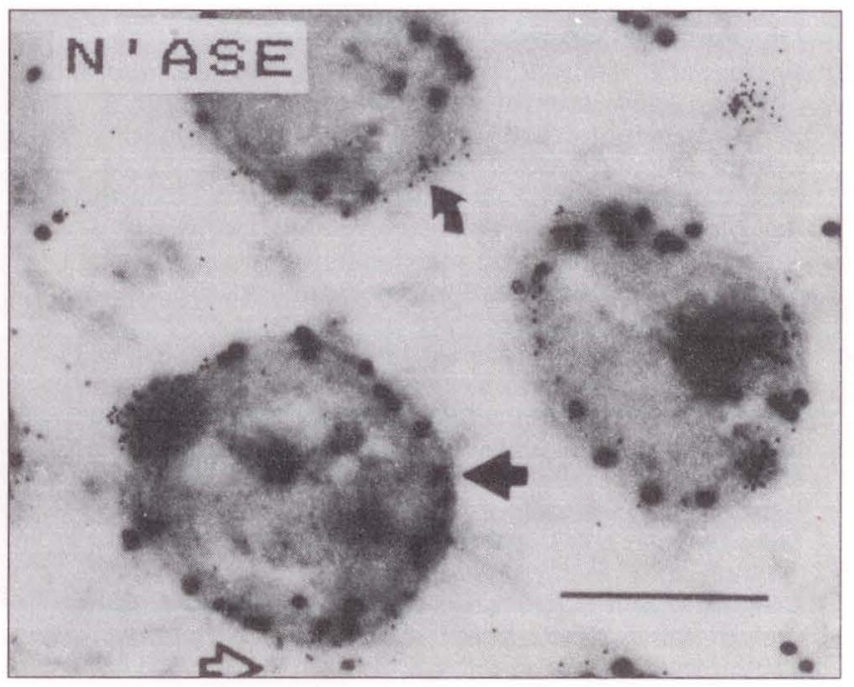

Figure 6b) Neuraminidase-treated urethral exudate. Very small gold particles indicated by the open arrows denote the initial reaction with the monoclonal antibody (MAb): this is minimal for the neuraminidase-treated exudate. The very large particles indicated by the straight solid arrows show that the cocci are gonococci by reaction with a specific antiserum. The intermediate sized particles indicated by the curved solid arrows show that the reaction with the MAb has been increased after incuba tion with neuraminidase 
TABLE 3

Neuraminidase/urethral exudate gonococci

\begin{tabular}{ccccc}
\hline Exudate & $\begin{array}{c}\text { No subculture } \\
\text { (\% resistance) }\end{array}$ & \multicolumn{2}{c}{$\begin{array}{c}\text { Subculture } \\
\text { (\% resistance) }\end{array}$} \\
\hline 1 & $>95$ & 13 & $<5$ & $>95$ \\
2 & $>95$ & 7 & $<5$ & $>95$ \\
3 & $>95$ & 18 & $<5$ & $>95$ \\
\hline
\end{tabular}

tion with the MAb after incubation either with medium or neuraminidase. Clearly, the reaction with the MAb has been increased by neuraminidase treatment, thus showing that gonococcal LPS in vivo is sialylated. At least three other urethral studies showed the same result. So the evidence from Buffalo is clearly that the LPS of the gonococcus is sialylated in vivo.

Now evidence from Birmingham. Let me first remind you of what I said at the start - gonococci in urethral exudates are resistant to killing by human serum and that the majority of them lose that resistance on one subculture. If this resistance is due to sialylation of the LPS, treatment of gonococci from patients with neuraminidase should change resistance into susceptibility. We had 11 samples of urethral exudates over the past eight months. In three of the samples there were not enough gonococci for meaningful experiments. Table 3 gives the results representative of those from the eight remaining samples. In contrast to controls (incubated with medium), there is a gross reduction in serum

\section{REFERENCES}

1. Rittenberg SC, Penn CW, Parsons NJ, Veale DR, Smith H. Phenotypic changes in the resistance of Neisseria gonorrhoeae to killing by normal human serum. J Gen Microbiol 1977; 103:69-75.

2. Martin PMV, Patel PV, Parsons NJ, Smith H. Induction of phenotypically determined resistance of Neisseria gonorrhoeae to human serum by factors in human serum. J Gen Microbiol 1981;127:213-7.

3. Patel PV, Martin PMV, Goldner M, Parsons NJ, Smith H. Red blood cells, a source of factors which induce Neisseria gonorrhoeae to resistance to complement-mediated killing by human serum. J Gen Microbiol 1984;130:2767-70.

4. Parsons NJ, Patel PV, Martin PMV, Goldner M, Smith H Gonococci in vitro and in vivo: Studies of the host and bacterial determinants of gonococcal resistance to killing by human serum and by phagocytes. In: Schoolnik GK, Brookes GF, Falkow S, et al, eds. The Pathogenic Neisseriae. Washington: American Society for Microbiology, 1985:487-94.

5. Parsons NJ, Constantinidou C, Todd B, Sammons CC, Cole JA, Smith H. Gonococci in most urethral exudates are sialylated: Neuraminidase changes serum resistance to susceptibility. In: Achtman M, Kohl P, Marchal C, Morelli G, Seiler A, Thiesen B, eds. Neisseriae 1990. Berlin: Walter de Gruyter, 1991:365-72.

6. Martin PMV, Patel PV, Parsons NJ, Smith H. Induction in gonococci of phenotypic resistance to killing by human serum by human genital secretions. Br J Vener Dis 1982;58:363-5.

7. Martin PMV, Patel PV, Clay JC, Parsons NJ, Smith H. Induction by human serum of resistance to serum in Neisseria gonorrhoeae: A clinical survey of patients with resistance of the in vivo-grown gonococci after treatment with neuraminidase without subculture. As reported in the past, these gonococci, once subcultured, were completely susceptible. After $3 \mathrm{~h}$ with CMPNANA to put the sialyl group back on, however, the resistance was restored to that of the gonococci in the urethral exudate only three or four days earlier (remember it is from different patients over an eight-month period). I think it is quite clear-cut evidence that the LPS of the gonococci in vivo is sialylated and that this action is responsible for the serum resistance. Hence, the evidence from Birmingham and Buffalo agrees.

The conclusions of the work are that phenotypic resistance in gonococci in vivo is due to sialylation of LPS, which inhibits reaction of bactericidal IgM with its target sites. The site of sialylation is the Gal $\beta 14 \mathrm{Glc}$ NAc terminal structure on the conserved LPS component of $4.5 \mathrm{kDa}$. The host product responsible is CMP-NANA, a substrate for sialyltransferase. When sialylation occurs, a surface coat of acid polysaccharide is seen. We still have to find out the connection between the sialylated component and the surface structure. In this work, we have accomplished what I think should be done more and more in studies of pathogenicity. We have identified a host factor, CMP-NANA. We have shown that it produces an identified virulence determinant namely a sialylated LPS component - and it happens in vivo. We have been specific about the in vivo environment and I think that it is how things could go in the future.

gonorrhoea. Br J Vener Dis 1984;60:151-3.

8. Patel PV, Parsons NJ, Andrade JRC, et al. White blood cells including polymorphonuclear phagocytes contain a factor which induces gonococcal resistance to complement-mediated serum killing. FEMS Microbiol Lett 1988;50:173-6.

9. Nairn CA, Cole JA, Patel PV, Parsons NJ, Fox JE, Smith $\mathrm{H}$. Cytidine 5'-monophospho-N-acetylneuraminic acid or a related compound is the low-Mr factor from human red blood cells which induces gonococcal resistance to killing by human serum. J Gen Microbiol 1988; 134:3295-306.

10. Parsons NJ, Patel PV, Tan EL, et al. Cytidine, 5 'monophospho-N-acetylneuraminic acid and a low molecular weight factor from human blood cells induce lipopolysaccharide alteration in gonococci when conferring resistance to killing by human serum. Microb Pathog 1988:5:303-9.

11. Parsons NJ, Andrade JRC, Patel PV, Cole JA, Smith H. Sialylation of lipopolysaccharide and loss of absorption of bactericidal antibody during conversion of gonococci to serum resistance by cytidine 5'-monophosphoN-acetylneuraminic acid. Microb Pathog 1989;7:63-72.

12. Tan EL, Patel PV, Parsons NJ, Martin PMV, Smith H. Lipopolysaccharide alteration is associated with induced resistance of Neisseria gonorrhoeae to killing by human serum. J Gen Microbiol 1986;132:1407-13.

13. Mandrell RE, Lesse AJ, Sugai JV, et al. In vitro and in vivo modification of Neisseria gonorrhoeae lipo-oligosaccharide epitope structure by sialylation. J Exper Med 1990;171:1649-64.

14. Parsons NJ, Cole JA, Smith H. Resistance to human serum of gonococci in urethral exudates is reduced by neuraminidase. Proc R Soc Lond 1990;B241:3-5. 
15. Fox AJ, Jones DM, Scotland SM, et al. Serum-killing of meningococci and several other Gram-negative bacterial species is not decreased by incubating them with cytidine 5'monophospho-N-acetylneuraminic acid. Microbiol Path 1989;7:317-8.

16. Fox AJ, Curry A, Rowland PL, et al. A surface polysaccharide forms when gonococci are converted to serum resistance by cytidine 5'monophospho$\mathrm{N}$-acetylneuraminic acid. FEMS Microbiol Lett 1990;66:75-80.
17. Fox AJ, Curry A, Jones DM, et al. The surface polysaccharide seen on Neisseria gonorrhoeae after treatment with CMP-NANA contains sialyl groups. In: Achtman M, Kohl P, Marchal C, Morelli G, Seiler A, Thiesen B, eds. Neisseriae 1990. Berlin: Walter de Gruyter, 1991:349-51.

18. Fox AJ, Curry A, Jones DM, et al. The surface structure seen on gonococci after treatment with CMP-NANA is due to sialylation of surface lipopolysaccharide previously described as a 'capsule'. Microbiol Path 1991;11:199-210. 


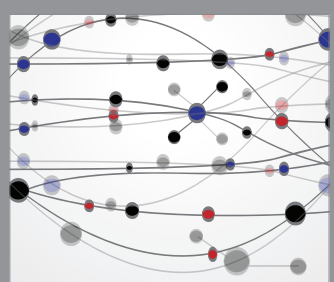

The Scientific World Journal
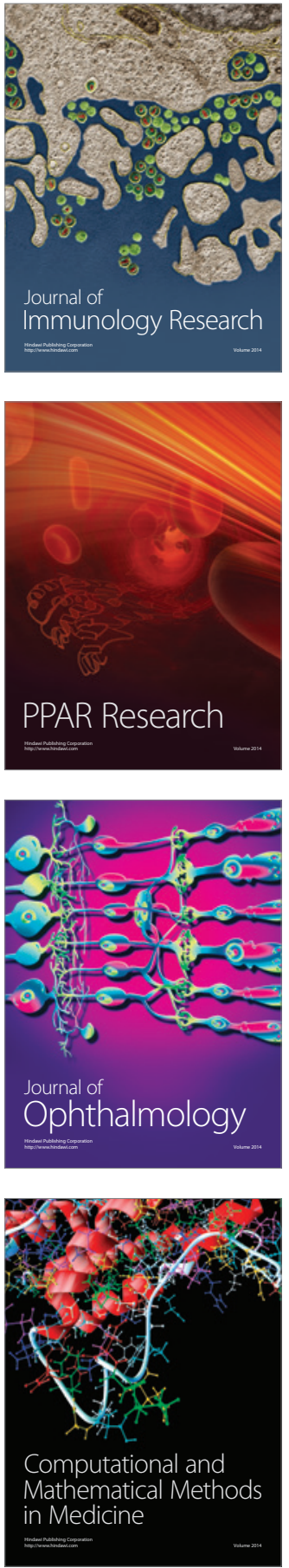

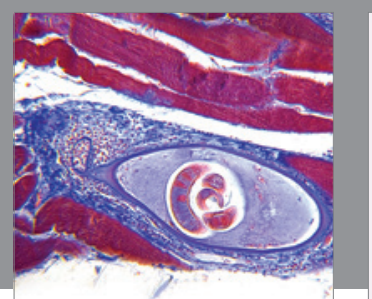

Gastroenterology Research and Practice

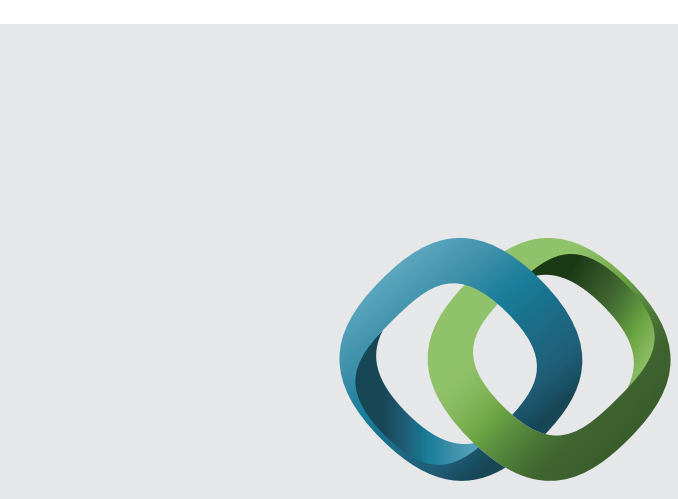

\section{Hindawi}

Submit your manuscripts at

http://www.hindawi.com
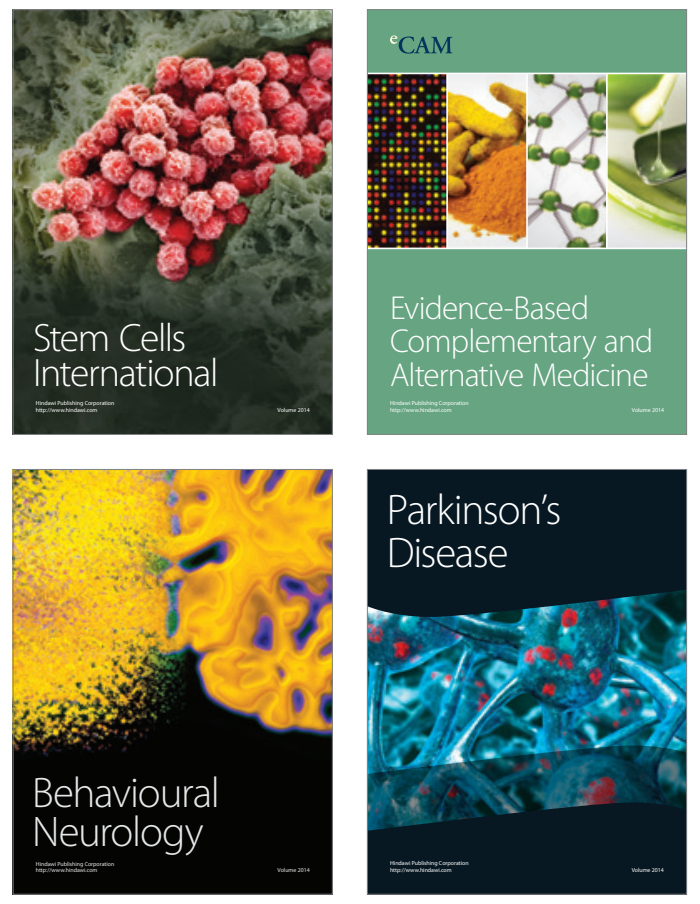
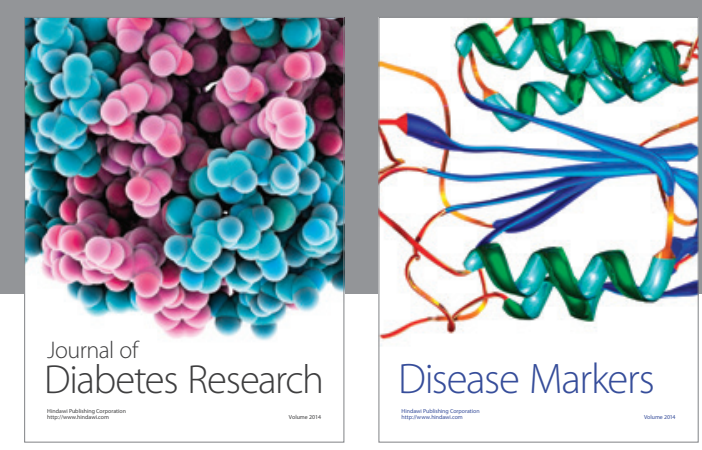

Disease Markers
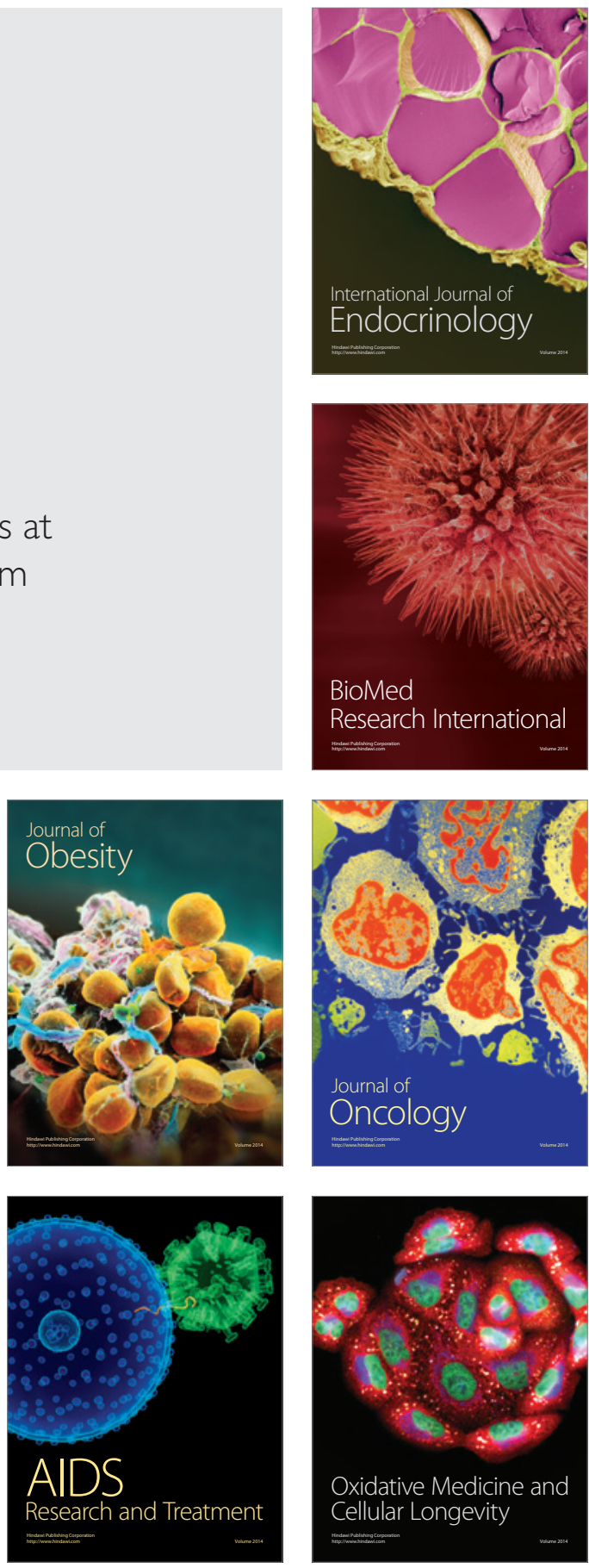